\title{
Digital Video Holography- A Robust TOOL FOR COMMUNICATION
}

\author{
S.Ponchitra ${ }^{1}$, K.Murali $^{1}$ and P.K.Palanisamy ${ }^{1}$ \\ ${ }^{1}$ Department of Physics, Anna University, Chennai \\ ponchitra@rocketmail.com
}

\begin{abstract}
Holograms are being produced using optical methods for decades. A lot of techniques and methods exist for the production of efficient holograms. Digital Holography (DH) is the method of simulating holograms with the use of computer. In this paper digital holograms are generated using Fresnel and Fraunhofer diffraction integrals. Multi color holograms are simulated and the digitally generated holograms are analysed. DH technique is extended further to video format which yields video holograms. The concept that every bit of a hologram contains full information of the original video, which is being effectively utilized to reduce the file size required for communication in terms of storage, security and speed. The entire process is simulated using Matlab7.10 environment.
\end{abstract}

\section{KEYWORDS}

Digital image holography, Digital video holography.

\section{INTRODUCTION}

Holography is a method of generating a three dimensional image of the object. It is done by recording the amplitude and phase of the object. Digital holography is the digital recording and numerical reconstruction of holographic data. Digital holography also includes optical construction and digital reconstruction [1] of data as well as digital construction and optical reconstruction [2] of data. In this paper, both the construction and reconstruction of hologram are done in digital domain with the aid of mathematical models.

Fresnel and Fraunhofer holograms have been generated using the diffraction integrals [1]. Several methods have been proposed for the recording of color holograms. Multi wavelength recording is used for the creation of color holograms. Pietro Ferraro et al. [3] and Li-Chien Lin [4] have used a color correction algorithm to avoid chromatic aberrations. Takashi Kakue et al [ 5,6 ] have incorporated phase shifting methods for the creation of color holograms. Han-Yen Tu et al have proposed GPU-based DH for three dimensional object recognition [7]. Also multiple SLMs and LEDs illumination have been used for colour holographic reconstruction [8]. Holographic compression has been developed for years and been proved as effective compression technique. Compression standards and Wavelet-bandelet transforms are incorporated in holographic data and they have resulted in a higher compression ratio $[9,10,11]$. In these works, a high compression ratio with very low NRMS error has been achieved by eliminating the spatial and temporal redundancies. All these methods are partially digital and partially optical. Also an algorithm or a complex manipulation is required for the compression of holographic data.

Sundarapandian et al. (Eds): CoNeCo,WiMo, NLP, CRYPSIS, ICAIT, ICDIP, ITCSE, CS \& IT 07, pp. 307-316, 2012. (C) CS \& IT-CSCP 2012

DOI : $10.5121 /$ csit.2012.2429 
This paper explains the method of generating multicolour holograms without the aid of any complex /correction algorithms and specialised setup. The interesting property of hologram is that the object information is flooded over the entire image plane hence each and every part of the hologram posses complete information of the object. This property is utilised to make hologram an effective tool for communication. A simple and time saving method for image/video processing in communication has been explained.

\section{DigitAL HOLOGRAPHY PROCESS}

Digital hologram can be generated for images in bmp, jpeg, tiff, png and gif image formats. The process involved in the generation of $\mathrm{DH}$ can be pointed out as

- Definition of object and reference

- Calculation of far field amplitude

- Construction of hologram

- Reconstruction of hologram

\subsection{Definition of Object and Reference}

Color holography, in optical sense is a superposition of holograms recorded with three different wavelengths. In digital holography since image is itself decomposed into three layers only one reference beam is enough to record color hologram. The Figure 1 shows the layers of the image that is taken into consideration. The sample object is shown in the top layer of Figure 1 as well as in Figure 2(a).

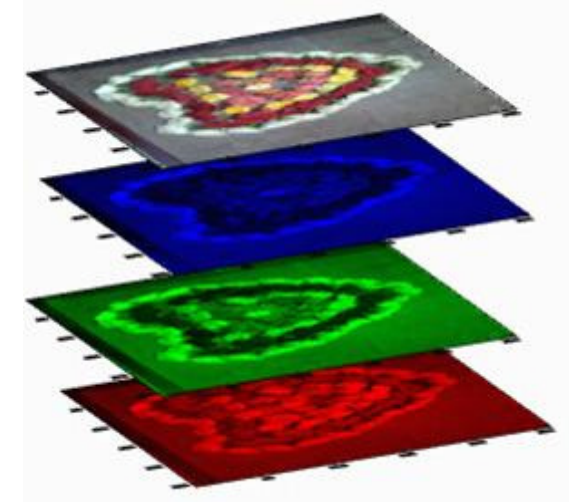

Figure 1. Layers of image using RGB colours.

The reference beam passes through the three layers of the object beam and hologram is generated by the superposition of three wavelengths with the reference beam. The reference beam originating from a point source is seen in Figure 2(b). An array of dimension similar to object beam is assigned as a reference beam with intensity values of zero in all the pixel locations except the centre pixel.

\subsection{Calculation of far field amplitude}

The far field amplitude calculation based on the Fraunhofer diffraction formula is the Fourier transform operation. The numerical equivalent of Fourier transform is Discrete Fourier Transform (DFT). The speed of computation is increased using Fast Fourier Transform (FFT). 


\subsection{Construction of hologram}

The hologram is the addition of far fields of the object and reference i.e. interference. The Fourier transform of the reference and the object matrices are added. The holographic equation thus calculated consists of terms which result in the creation of conjugate images along with the original reconstructed images. Hence filtering is done to extract the original image. The constructed hologram is shown in Figure 2(c).

\subsection{Reconstruction of hologram}

The constructed hologram can be reconstructed using Fresnel and Fraunhofer diffraction integral.

\section{4(a) Fraunhoffer method}

The matrix corresponding to the filtered hologram is Fourier transformed as per the diffraction equation [1]. The intensity of the resulting matrix is calculated and imaged. The reconstructed image is shown in Figure 2(d).

\section{4(b) Fresnel method}

The distance of separation between the hologram plane and the observation plane "d", pixel size " $\Delta \mathrm{m}$ " and " $\Delta \mathrm{n}$ " , wavelength " $\lambda$ " are chosen as, $\mathrm{d}=1 \mathrm{~m}, \Delta \mathrm{m}=6.8 \mathrm{~nm}, \Delta \mathrm{n}=6.8 \mathrm{~nm}$ and $\lambda=633 \mathrm{~nm}$. The discrete Fresnel transform given in equation is calculated as per the equation. The reconstructed image is shown in Figure 2(e). The image reconstructed using this method contains phase term. The numerical processing of Fresnel Kirchhoff diffraction integral is time consuming [3].

Image reconstructed using Fresnel diffraction contains the phase term. Since the image taken for study is two dimensional phase term does not play a role. Hence it is enough to continue with the Fraunhofer reconstruction.

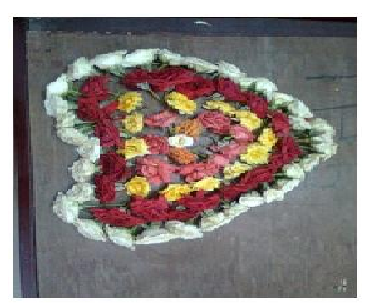

(a)

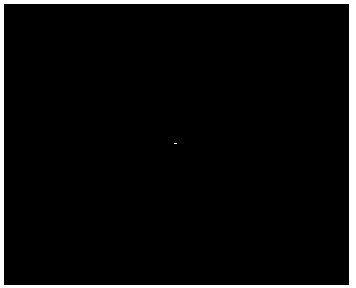

(b)

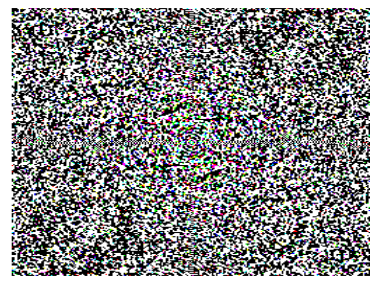

(c) 


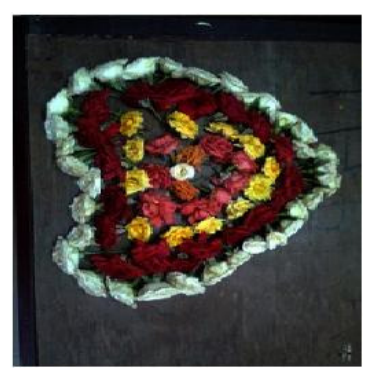

(d)

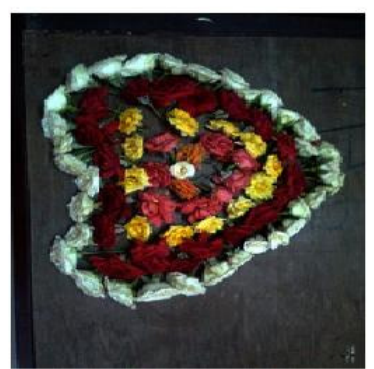

(e)

Figure 2 (a) Input image (b) Reference Image (c) Constructed digital hologram

(d) Reconstructed Image by Fraunhofer Method (e) Reconstructed Image by Fresnel Method

\section{Digital Video Holography}

Video refers to recording, manipulating and display of moving images. One particular still image is said to be a frame. Video is a stack of images moved one by one in a particular frame rate. When a moving picture is displayed, each frame is flashed on the screen for a short time and then immediately replaced by the next one. Video holography can be experienced by calculating the hologram for each and every frame in a particular video and reconstructing one by one [12]. Matlab can support any formats supported by Microsoft DirectShow. Hence, the holograms from different formats can also be calculated.

\subsection{Hologram Construction}

An object is created to read a video file. The Read only properties of the object are Bits per pixel, duration, frame rate, height, name, number of frames, path, tag, type, user data, video format and width. Variables are used to access the number of frames, height and width from the input video. Memory for the movie structure must be allotted initially in accordance with the height and width of the frame. Three video files are initialised for the object, hologram and the reconstructed video. The frames from the input video are extracted. The video is now visualised as „n number of images to be processed.

From the video frame, the pixel details are retrieved. The reference beam is initialized as in the case of image in holography.The hologram of the frame is computed and the intensity distribution of the computed hologram is added to a new video frame which is allotted initially. The process is executed for "n" number of frames of the input video. At the end of the frames, the movie is terminated and saved in the predefined path.

\subsection{Hologram Reconstruction}

As in image hologram, video can also be reconstructed using Fresnel and Fraunhofer method. In concern with the time consumption, Fraunhofer method is adopted in this paper. Also the input taken into consideration is of two dimensions. From the hologram video, individual frames are extracted and reconstructed. For individual frames, reconstructed matrix is calculated and is then appended to the pre allocated movie structure. 


\section{Analysis of Digital Hologram}

\subsection{Negative of a Hologram}

The hologram of interest is shown in Figure 3(a). The image is reconstructed as discussed in previous section. The negative of the hologram is calculated and is reconstructed. The negative of the hologram is shown in Figure 3(b). The image reconstructed from the original hologram and it"s negative are shown in Figures 3(c) and 3(d) respectively. From these figures it is evident that the hologram and its negative produce identical images.

In digital communication if hologram is used for the transmission of image/video, the bit reversal problem can not affect the transmission since both produces identical images on reconstruction. Hence the bit error rate during transmission can be reduced.

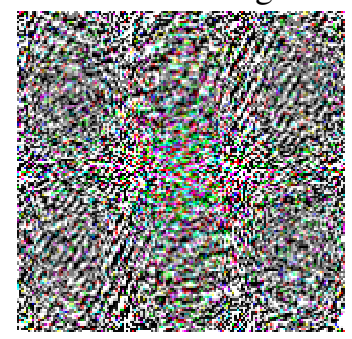

(a)

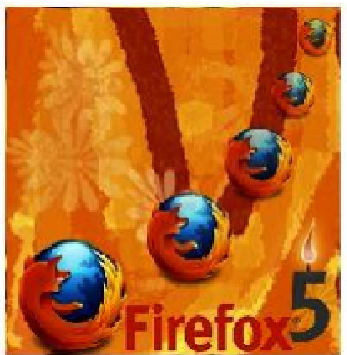

(c)

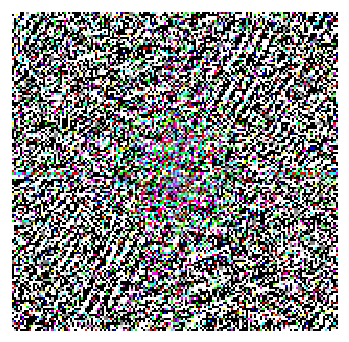

(b)

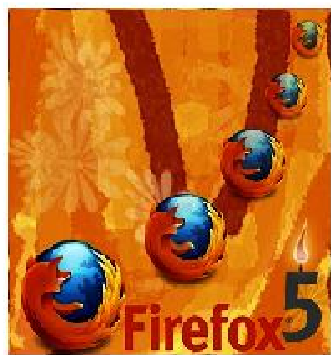

(d)

Figure 3 (a)Input Hologram (b) Negative of the Hologram (c) Reconstructed from the Input Hologram (d) Reconstructed from negative of the Hologram

\subsection{Reconstruction from various regions of the Hologram}

Hologram contains the information of the entire object at each and every point. This is

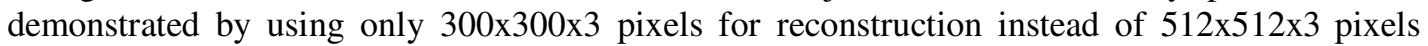
where $512 \times 512 \times 3$ is the pixel size of the input image. From the calculated hologram matrix, the top left portion is selected for reconstruction. Similarly, top right portion, centre, bottom left and bottom right portions of the hologram are selected and they are reconstructed individually. 


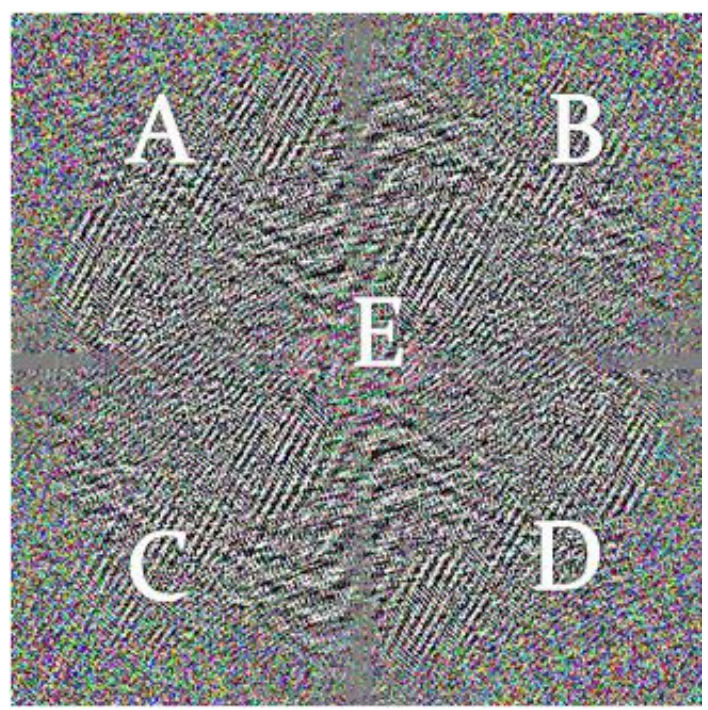

Figure 4 Input Hologram

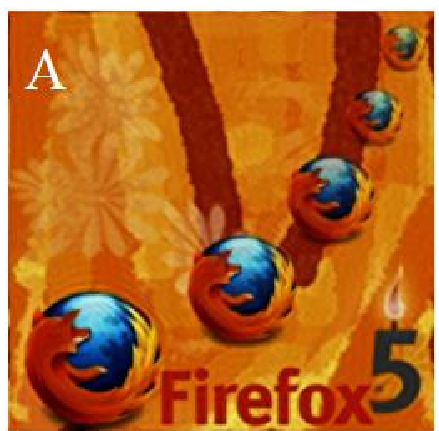

(a)

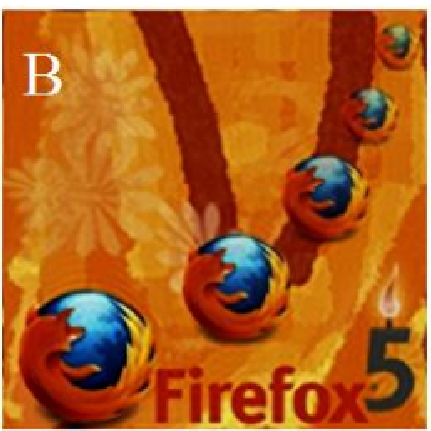

(b)

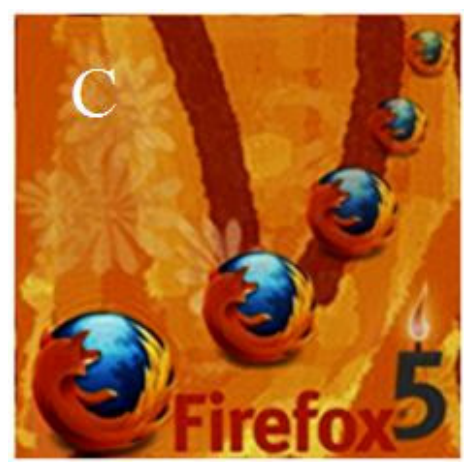

(c) 


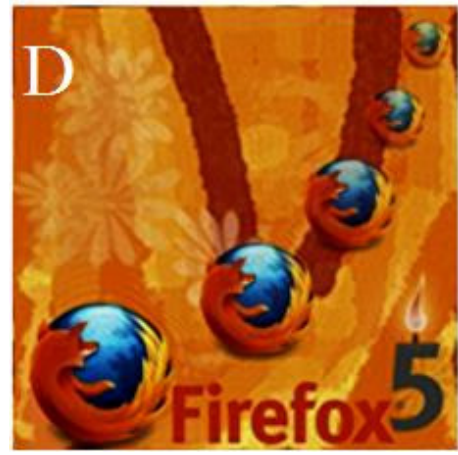

(d)

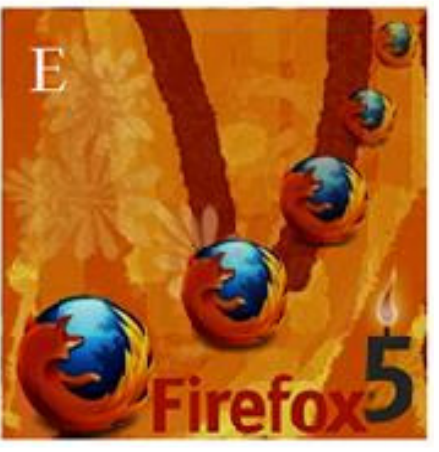

(e)

Figure 5 Reconstructed from the particular regions of the hologram as indicated with the alphabets

Just for reference alphabets are posted in the figure. The reduction in the number of pixels used for reconstruction leads to reduction in the resolution of the reconstructed image. Image reconstructed from the low frequency components of the hologram produces a smooth image compared to the reconstruction from the high frequency components. Hologram of $512 \times 512 \times 3$ image is generated. Reconstruction done using $100 \times 100 \times 3$ pixel values from the top portion of the hologram is shown in Fig 6(a) and image reconstructed using central 100x100x3 pixels is shown in Fig 6(b). For reconstruction from high frequency components the minimum number of pixels required for reconstruction is $\mathrm{N} / 2 \mathrm{xN} / 2 \times 3$ whereas in case of low frequency components even with $100 \times 100 \times 3$ pixels good quality image is reconstructed.

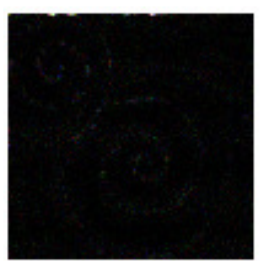

(a)

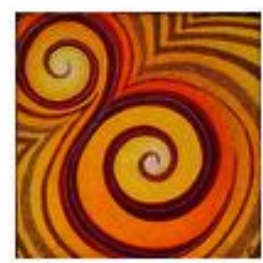

(b)

Fig6 Reconstructed from (a) top portion of the hologram. (b) central portion of the hologram

Table 1. Reducing the storage memory size of the images using holograms

\begin{tabular}{|c|c|c|}
\hline Pixel size used for Reconstruction & Memory size & Reconstructed Image \\
\hline $100 \times 100 x 3$ & $4.26 \mathrm{~KB}$ & \\
\hline $50 \times 50 \times 3$ & $1.81 \mathrm{~KB}$ & (ด) \\
\hline $10 \times 10 \times 3$ & 703 Bytes & 娄 \\
\hline
\end{tabular}


Table1 shows the pixel size used for reconstruction with its corresponding reconstructed image size. Even a 10x10x3 pixels from the hologram can be utilized to reconstruct the entire image but with low resolution. If the entire $512 \times 512 \times 3$ hologram is used for reconstruction, the reconstructed image occupies a memory space of $42.8 \mathrm{~KB}$. In case of $256 \times 256 \times 3$ pixels used, memory space is $15.9 \mathrm{~KB}$. Thus the hologram can be realised as a lossy compression tool where the amount of compression can be altered depending on the application. Thus flexibility in the amount of compression can be achieved using digital holography.

The same process can be experienced for a video file. The height and width of the original frame in the video under study is 240 and 320 . Hence the still image of the video is an array of dimension 240x320x3 and so will be the calculated hologram matrix. From this array, portion of the image is cropped and the cropped images are stacked as a new video. With this video as input, the object beam is reconstructed. The size of the input video file used in this work is 30.9 MB. A portion of $140 \times 180 \times 3$ is chosen from the hologram video. Upon reconstruction, the size of the video is $11.3 \mathrm{MB}$. A compression ratio of $36.57 \%$ is achieved in this cropping which yields a space savings of $63.43 \%$.

During transmission of image/video, edges are more sensitive to noise compared to other low frequency components. Hence if holographic transmission comes into play, even if the edges got affected by noise the low frequency components are enough to reconstruct the entire image / video as explained previously. This paves the way for holography to view as a robust communication. Also the hologram cannot be reconstructed without the knowledge of the reference beam used in the construction phase hence it forms a secured mode of communication.

\subsection{Data Transfer Time}

Data transfer time is the time taken for a digital data to move from one place to another. It can be viewed as the speed of travel of a given amount of data from one place to another. In telecommunication, data transfer is usually measured in bits per second. The Table 2 shows the time taken for the video transmission under different transfer rates. For the raw input video file and the compressed video file taken into consideration, the data transfer time is calculated using the calc tool [13].

Table 2. Data transfer time for different transfer rates

\begin{tabular}{|l|l|l|}
\hline Speed/transfer rate & $\begin{array}{l}\text { Data Transfer time before } \\
\text { compression }\end{array}$ & $\begin{array}{l}\text { Data Transfer time after } \\
\text { compression }\end{array}$ \\
\hline $512 \mathrm{Kbps}$ & 506.27 seconds & 185.14 seconds \\
\hline $155.52 \mathrm{Mbps}$ OC3 & 1.67 seconds & 0.61 seconds \\
\hline $622.08 \mathrm{MbpsOC12}$ & 0.42 seconds & 0.15 seconds \\
\hline
\end{tabular}


Though the actual transfer speed is slower than the calculated time due to latency, jitter, speed of the modem and overhead, but still an efficient space saving has been achieved using the holographic video compression.

\section{CONCLUSIONS}

Digital holography is used to reduce the size of the image/video file. It is used to resize, compress and optimise the image and video files in order to make them compatible for web pages, email attachments and disk storage. DH can be used as a model for robust, secured and efficient communication without use of any additional algorithms and hence the power and time.A three dimensional color image of a person in remote location can be generated and this can be used for commercial displays.

\section{REFERENCES}

[1] Ulf Schnars and Werner P O Juptner, "Digital recording and numerical reconstruction of holograms", Institute of Physics Publishing, 17 July 2002.

[2] Pedro Pombo, Ângelo Arrifano , Joao Lemos Pinto," Computer generated binary Fourier holograms", Proceedings of SPIE,2006.

[3] Pietro Ferraro, Simonetta Grilli,, Lisa Miccio, Domenico Alfieri ,Sergio De Nicola, Andrea Finizio, and Bahram Javidi, "Full Color 3-D Imaging by Digital Holography and Removal of Chromatic Aberrations", Journal of Display Technology, Vol. 4, No. 1, March 2008

[4] Li-Chien Lin "Correction of Chromatic distortion for Color Digital Holographic system", IEEE Transactions, 2011.

[5] Takashi Kakue, Tatsuki Tahara, Kenichi Ito, Yuki Shimozato, Yasuhiro Awatsuji, Kenzo Nishio, Shogo Ura, Toshihiro Kubota, and Osamu Matoba "Numerical verification of single-shot two- step phase-shifting color digital holography”, IEEE Transactions, 2009.

[6] Yasuhiro Awatsuji, Takamasa Koyama, Atsushi Kaneko, Atsushi Fujii, Kenzo Nishio, Shogo Ura, And, Toshihiro Kubota "Single-shot phase-shifting color digital holography", IEEE Transactions, 2007.

[7] Han-Yen Tu, Yi-Ta Lee, and Chau-Jern Cheng, "GPU-based Digital Holography for ThreeDimensional Color Object Recognition”,IEEE Transactions, 2011.

[8] Fahri Yara s, Levent Onural "Color Holographic Reconstruction Using Multiple SLMs and LED Illumination", Proceedings of SPIE-IS\&T Electronic Imaging, SPIE Vol. 7237, 2009.

[9] Le Thanh Bang, Zulfiqar Ali, Pham Duc Quang, Jae-Hyeung Park, Nam Kim, "Compression of digital hologram for three dimensional object using Wavelet-Bandelets Transform”, Optics Express, 2011

[10] Emmanouil Darakis a and Thomas J. Naughton" Compression of digital hologram sequences using MPEG-4", Proceedings of SPIE, 2009

[11] Emmanouil Darakis, Marcin Kowiel, Risto Nasanena and Thomas J. Naughton, "Visually lossless compression of digital hologram sequences", Proceedings of SPIE, 2011

[12] http://www.mathworks.in/help/techdoc/ref/videoreaderclass.html

[13] http://www.t1shopper.com/tools/calculate/downloadcalculator.php 


\section{Authors}

\section{Short Biography}

P.K.Palanisamy joined Anna University, Chennai as a faculty in Department of Physics in 1979 and presently he is working as Professor of Eminence. He obtained his Ph.D. degree in Laser Technology from Anna University in 1990. His field of current research interests are Photonics, Holography and nano science.

K.Murali obtained his Ph.D. degree in nonlinear dynamics in 1995 from Bharathidasan University, Tiruchirapalli. In 1998 he joined as a faculty in Department of Physics, Anna University, Chennai - 600 025 and presently, he is working as Associate Professor. His field of current research interests are Nonlinear dynamics, Chaos Theory \& Applications, Nonlinear optics, Nano and power electronics.

S.Ponchitra joined Anna University, Chennai as a student in Faculty of Information and Communication Engineering(Masters in Laser and Electro Optical Engineering) in 2010. She obtained her Bachelor degree in department of ECE in 2010. Her field of research interest is Holography. 